\section{Rule Out (R/O) Arteriovenous Malformation}

When imaging patients for intracranial arteriovenous malformations, the goals are (1) to determine if an arteriovenous malformation is present; (2) to assess size, shape, and location of the nidus; (3) to determine potential arterial feeders and venous drainage routes (including screening for aneurysms on arterial feeders); and (4) to determine the state of the surrounding brain parenchyma, including the identification of intracranial hemorrhage. A post-contrast 3-D spoiled gradient echo (SPGR) sequence is used for sequences 1 to 3 and the standard MR imaging sequences are used to evaluate the brain parenchyma. The following Basic Protocol can be used for the evaluation of stable patients.

In order to perform the optional sequences with perfusion and diffusion studies, a scanner with echoplanar capabilities is required (Table A1.5.1). The standard anatomical MR imaging sequences included in the protocols do not require these faster gradients. The parameters given here are optimized for a 1.5T GE LX system with 11.0 software and may need modification for different software versions, field strengths, or manufacturers.

NOTE: Be sure that technologists and nurses have immediate access to any emergency equipment that may be relevant to a given study, or that may be needed for a particular patient, such as crash carts or oxygen. Reactions to contrast agents are rare, but the resources are necessary.

\section{Materials}

Gadolinium-based MR contrast agent (e.g., Magnevist, Omniscan, Prohance)

Normal saline $(0.9 \% \mathrm{NaCl})$, sterile

Table A1.5.1 Equipment Requirements for Cerebral Vascular Assessment

\begin{tabular}{ll}
\hline Type of system & LX EchoSpeed \\
Field strength & $1.5 \mathrm{~T}$ \\
Software level & 11 \\
Magnet type & CX K4 \\
Polarity & Positive \\
Active gradient shielding & No \\
Passive gradient shielding & No \\
Gradient strength (amplitude) & $2.4 \mathrm{mT} / \mathrm{cm}$ \\
Slew rate & $12 \mathrm{mgauss} / \mathrm{cm}$ \\
SAR (average) & $2 \mathrm{~W} / \mathrm{kg}$ \\
SAR (max) & $8 \mathrm{~W} / \mathrm{kg}$ \\
Superior conductive shimming & $45 \mathrm{~cm}$ \\
Resistive shimming & No \\
Active magnetic shielding & No \\
Maximum noise level & $100 \mathrm{~dB}$ \\
Type of body coil & High pass \\
TPS recon system memory & 386 \\
Array processor (TPS) & Reflex 100 \\
Computer & LX Octane \\
Weight limit & $350 \mathrm{lb}(130.6 \mathrm{~kg})$ \\
\hline
\end{tabular}

UNIT A1.5

$B A S I C$

PROTOCOL
Intracranial Arterial Disease

\section{A1.5.1}

Supplement 13 


\section{Set up patient and equipment}

1. Interview (screen) the patient to ensure that he or she has no contraindications such as cardiac pacemakers or other implants containing ferromagnetic materials. Also be sure to find out if the patient has any health conditions that may require the presence of special emergency equipment during the scanning procedure, or necessitate any other precautions. Do not forget to ask if the patient has any drug allergies and document them.

Generally, standard screening forms are used for all patients scanned in a magnetic resonance system (see APPENDIX 1).

The presence of any ferromagnetic metals may be a health hazard to the patient when he or she is inside the magnet, and will also affect the imaging. If in doubt as to the exact composition of the items, it is best to exclude patients with any metal implants; see Shellock (1996) for discussion of what implants may be safely scanned using magnetic resonance.

Patients may be accompanied into the magnet room by a friend or family member, who can sit in the room during the scan and comfort the patient as needed. This companion must be screened as well to ensure the absence of loose metal objects on the body or clothing.

2. If the procedure is a research protocol, have the patient sign any necessary consent form.

3. Have the patient remove all jewelry and change into a gown to eliminate any metal that might be found in clothing.

4. Have the patient wash off any mascara and other makeup to avoid local tissue heating and image artifacts.

5. Inform the patient about what will occur during the procedure, what he or she will experience while in the magnet, and how to behave, including the following:

a. If earphones or headphones are used to protect the ears from the loud sounds produced by the gradients, the patient will be asked to wear these, but will be able to communicate with the technologist at any/all times during the imaging.

b. The patient will be given a safety squeeze-bulb or similar equipment to request assistance at any time (demonstrate how this works).

c. For optimum results the patient should not talk, and should avoid or minimize swallowing or other movement, during each scan-i.e., as long as the banging sounds continue. Between scans, talking and swallowing are allowed in most cases, but should be avoided when comparative positional studies are being performed; the patient will be informed when this is the case.

d. Nevertheless, the patient may call out at any time if he or she feels it necessary.

6. Have the patient mount onto the table. Either before or right after the patient lies down, set up any triggering devices or other monitoring equipment that is to be used.

7. Establish an intravenous line from which the contrast agent can be injected, and attach this line securely to the patient so that movement into or out of the magnet will not pull at the patient's arm.

It is preferable to insert the line prior to imaging and to leave the patient in the magnet, so that there is no intervening motion between the scans run before contrast agent injection and those run after injection. 
8. Center the patient in a head or neck coil at the region where the key information is desired. Make sure that the head and neck are constrained to prevent motion, especially if high-resolution scans are to be run.

Generally the patient's head is fixed so that the head is horizontal (not tilted) and the neck and head lie along the axis of the patient table; other positions may be appropriate depending on the needs at hand.

Most scanners have a special neck coil for MRA; otherwise, a head coil should be used and the patient placed as far in as possible so that the bifurcation of the main carotid artery into the internal and external carotid arteries can be imaged.

9. If needed, place a pillow or other support under the knees to make the patient more comfortable.

10. Use the centering light to position the patient and put him or her into the center of the magnet.

Once this step has been performed, so long as the patient does not move on the table, the table itself can be moved and then replaced in the same position as before without jeopardizing the positioning of one scan relative to another.

11. If the patient is unable to hold still, provide an appropriate sedative.

12. Have a single dose of 0.1 to $0.2 \mathrm{mmol} / \mathrm{kg}$ of contrast agent drawn up and ready for injection. Have an angiocatheter or butterfly, alcohol swabs, and tourniquet ready.

\section{Sequence 1: Rapid sagittal $T_{1}$-weighted scout}

13. To determine the patient's position, perform a fast sagittal scout scan using the imaging sequence in Table A1.5.2.

Some centers may prefer a rapid three-plane scout instead of this multislice sagittal scout.

Table A1.5.2 Rapid Sagittal $T_{1}$-Weighted Scout

\begin{tabular}{ll}
\hline Patient position & Supine \\
Scan type & Gradient echo \\
Imaging plane (orientation) & Sagittal \\
Central slice or volume center & Nasion \\
Echo time $\left(T_{\mathrm{E}}\right)$ & Minimum (at least $8.2 \mathrm{msec})$ \\
Receiver bandwidth $(\mathrm{RBW})$ & $15.6 \mathrm{kHz}$ \\
Repeat time $\left(T_{\mathrm{R}}\right)$ & $100 \mathrm{msec}$ \\
Flip angle $(\mathrm{FA})$ & $60^{\circ}$ \\
Fields of view $\left(\mathrm{FOV}_{\mathrm{x}}, \mathrm{FOV}\right.$ & $\mathrm{y})$ \\
Resolution $(\Delta x, \Delta y)$ & $240 \mathrm{~mm}, 240 \mathrm{~mm}$ \\
Number of data points collected $\left(N_{\mathrm{x}}, N_{\mathrm{y}}\right)$ & $0.94 \mathrm{~mm}, 1.87 \mathrm{~mm}$ \\
Display matrix $\left(D_{\mathrm{x}}, D_{\mathrm{y}}\right)$ & 256,128 \\
Slice thickness $(\Delta z)$ & 128,128 \\
Number of slices & $7 \mathrm{~mm}$ \\
Slice gap & 7 \\
Number of excitations $(\mathrm{NEX})$ & $2 \mathrm{~mm}$ \\
Number of acquisitions $\left(N_{\mathrm{acq}}\right)$ & 1 \\
Read direction & 1 \\
Scan time & Anterior-posterior \\
\hline
\end{tabular}


Table A1.5.3 Transverse $T_{2}$-Weighted FSE

\begin{tabular}{ll}
\hline Patient position & Supine \\
Scan type & Fast spin echo XL \\
Imaging plane (orientation) & Transverse \\
Variable bandwidth & Yes \\
Pulse sequence database (PSD) & FSE-XL \\
Central slice or volume center & Nasion \\
Echo time $\left(T_{\mathrm{E}}\right)$ & $102 \mathrm{msec}$ \\
Receiver bandwidth $(\mathrm{RBW})$ & $11.36 \mathrm{kHz}$ \\
Echo train length $(\mathrm{ETL})$ & 12 \\
Repeat time $\left(T_{\mathrm{R}}\right)$ & $6000 \mathrm{msec}$ \\
Flip angle $(\mathrm{FA})$ & $90^{\circ}$ \\
Fields of view $(\mathrm{FOV}, \mathrm{x}, \mathrm{FOV}$ & $\mathrm{y})$ \\
Resolution $(\Delta x, \Delta y)$ & $220 \mathrm{~mm}, 165 \mathrm{~mm}$ \\
Number of data points collected $\left(N_{\mathrm{x}}, N_{\mathrm{y}}\right)$ & $0.86 \mathrm{~mm}, 0.86 \mathrm{~mm}$ \\
Display matrix $\left(D_{\mathrm{x}}, D_{\mathrm{y}}\right)$ & 256,192 \\
Slice thickness $(\Delta z)$ & 320,256 \\
Number of slices & $5 \mathrm{~mm}$ \\
Slice gap & 24 \\
Number of excitations $(\mathrm{NEX})$ & $1 \mathrm{~mm}$ \\
Number of acquisitions $\left(N_{\mathrm{acq}}\right)$ & 2 \\
Read direction & 1 \\
Flow compensation & Anterior-posterior \\
Extended dynamic range $(\mathrm{EDR})$ & Yes \\
Spatial saturation & Yes \\
Scan time & Inferior \\
\hline
\end{tabular}

Sequence 2: Transverse $T_{2}$-weighted fast spin echo (FSE)

14. From the sagittal scout, select the image through the center of the brain to set up the locations for the transverse $T_{2}$-weighted fast spin echo (FSE) sequence. Set up the imaging parameters as shown in Table A1.5.3.

The locations should be the same as those chosen for the transverse diffusion.

15. Warn the patient that this sequence is starting and begin the scan.

\section{Sequence 3: Transverse fluid-attenuated inversion recovery (FLAIR)}

16. From the sagittal scout, select the image through the center of the brain to set up the locations for the transverse fluid-attenuated inversion recovery (FLAIR). Set up the imaging parameters as shown in Table A1.5.4.

The locations should be the same as those chosen for the FSE sequence.

17. Warn the patient that this sequence is starting and begin the scan.

\section{Sequence 4: Transverse $T_{2}$-weighted gradient echo}

18. To detect hemorrhage, from the sagittal scout select the image through the center of the brain to set up the locations for the transverse gradient echo $T_{2}$-weighted sequence. Set up the imaging parameters as shown in Table A1.5.5.

Rule Out (R/O)

Arteriovenous Malformation
The locations should be the same as those chosen for the FSE sequence.

19. Warn the patient that this sequence is starting and begin the scan. 
Table A1.5.4 Transverse FLAIR

\begin{tabular}{ll}
\hline Patient Position & Supine \\
Scan type & Inversion recovery, FSE \\
Variable bandwidth & Yes \\
Imaging plane (orientation) & Transverse \\
Central slice or volume center & Nasion \\
Echo time $\left(T_{\mathrm{E}}\right)$ & $120 \mathrm{msec}$ \\
Receiver bandwidth $(\mathrm{RBW})$ & $20.83 \mathrm{kHz}$ \\
Repeat time $\left(T_{\mathrm{R}}\right)$ & $10,000 \mathrm{msec}$ \\
Inversion time $\left(T_{\mathrm{I}}\right)$ & $2200 \mathrm{msec}$ \\
Flip angle $(\mathrm{FA})$ & $90^{\circ}$ \\
Fields of view $(\mathrm{FOV}, \mathrm{FOV}$ \\
Resolution $(\Delta x, \Delta y)$ & $220 \mathrm{~mm}, 220 \mathrm{~mm}$ \\
Number of data points collected $\left(N_{\mathrm{x}}, N_{\mathrm{y}}\right)$ & $0.86 \mathrm{~mm}, 1.14 \mathrm{~mm}$ \\
Display matrix $\left(D_{\mathrm{x}}, D_{\mathrm{y}}\right)$ & 256,192 \\
Slice thickness $(\Delta z)$ & 256,256 \\
Number of slices & $5 \mathrm{~mm}$ \\
Slice gap & 24 \\
Number of excitations $(\mathrm{NEX})$ & $1 \mathrm{~mm}$ \\
Number of acquisitions $\left(N_{\mathrm{acq}}\right)$ & 1 \\
Read direction & 2 \\
Saturation pulses & Inferior \\
Scan time & $4 \mathrm{~min}, 44 \mathrm{sec}$ \\
\hline
\end{tabular}

Table A1.5.5 Transverse $T_{2}$-Weighted Gradient Echo

\begin{tabular}{|c|c|}
\hline Patient position & Supine \\
\hline Scan type & Gradient echo \\
\hline Imaging plane (orientation) & Transverse \\
\hline Variable bandwidth & Yes \\
\hline Central slice or volume center & Nasion \\
\hline Echo time $\left(T_{\mathrm{E}}\right)$ & $25 \mathrm{msec}$ \\
\hline Receiver bandwidth (RBW) & $15.63 \mathrm{kHz}$ \\
\hline Repeat time $\left(T_{\mathrm{R}}\right)$ & $750 \mathrm{msec}$ \\
\hline Flip angle (FA) & $20^{\circ}$ \\
\hline Fields of view $\left(\mathrm{FOV}_{\mathrm{x}}, \mathrm{FOV}_{\mathrm{y}}\right)$ & $220 \mathrm{~mm}, 165 \mathrm{~mm}$ \\
\hline Resolution $(\Delta x, \Delta y)$ & $0.86 \mathrm{~mm}, 1.14 \mathrm{~mm}$ \\
\hline Number of data points collected $\left(N_{\mathrm{x}}, N_{\mathrm{y}}\right)$ & 256,144 \\
\hline Display matrix $\left(D_{\mathrm{x}}, D_{\mathrm{y}}\right)$ & 256,192 \\
\hline Slice thickness $(\Delta z)$ & $5 \mathrm{~mm}$ \\
\hline Number of slices & 24 \\
\hline Slice gap & $1 \mathrm{~mm}$ \\
\hline Number of excitations (NEX) & 2 \\
\hline Number of acquisitions ( $N_{\text {acq }}$ ) & 1 \\
\hline Read direction & Anterior-posterior \\
\hline Flow compensation & Yes \\
\hline Scan time & $3 \mathrm{~min}, 41 \mathrm{sec}$ \\
\hline
\end{tabular}


Table A1.5.6 Transverse $T_{1}$-Weighted Spin Echo

\begin{tabular}{ll}
\hline Patient position & Supine \\
Scan type & Spin echo \\
Imaging plane (orientation) & Transverse \\
Variable bandwidth & Yes \\
Central slice or volume center & Nasion \\
Echo time $\left(T_{\mathrm{E}}\right)$ & Minimum full $(14 \mathrm{msec})$ \\
Receiver bandwidth $(\mathrm{RBW})$ & $15.6 \mathrm{kHz}$ \\
Repeat time $\left(T_{\mathrm{R}}\right)$ & $400 \mathrm{msec}$ \\
Flip angle $(\mathrm{FA})$ & $90^{\circ}$ \\
Fields of view $(\mathrm{FOV}, \mathrm{FOV}$ & $\mathrm{y})$ \\
Resolution $(\Delta x, \Delta y)$ & $220 \mathrm{~mm}, 165 \mathrm{~mm}$ \\
Number of data points collected $\left(N_{\mathrm{x}}, N_{\mathrm{y}}\right)$ & $0.86 \mathrm{~mm}, 1.14 \mathrm{~mm}$ \\
Display matrix $\left(D_{\mathrm{x}}, D_{\mathrm{y}}\right)$ & 256,144 \\
Slice thickness $(\Delta z)$ & 256,192 \\
Number of slices & $5 \mathrm{~mm}$ \\
Slice gap & 24 \\
Number of excitations $(\mathrm{NEX})$ & $1 \mathrm{~mm}$ \\
Number of acquisitions $\left(N_{\mathrm{acq}}\right)$ & 1 \\
Read direction & 2 \\
Flow compensation & Anterior-posterior \\
Extended dynamic range $(\mathrm{EDR})$ & No \\
Saturation pulses & Yes \\
Scan time & Inferior \\
\hline
\end{tabular}

\section{Sequence 5: Transverse $T_{1}$-weighted spin echo}

20. From the sagittal scout, select the image through the center of the brain to set up the locations for the transverse $T_{1}$-weighted spin echo sequence. Set up the imaging parameters as shown in Table A1.5.6.

The locations should be the same as those chosen for the transverse diffusion.

21. Warn the patient that this sequence is starting and begin the scan.

\section{Sequence 6: Transverse post-contrast 3-D spoiled gradient echo}

22. Let the patient know that you are going to bring them out of the scanner and give them the contrast injection.

23. Bring the patient out of the scanner and inject the single dose of contrast agent intravenously.

24. From the sagittal scout, select the image through the center of the brain to set up the locations for the transverse 3-D spoiled gradient echo sequence. Set up the imaging parameters as shown in Table A1.5.7.

The locations should be the same as those chosen for the FSE sequence.

25. Warn the patient that this sequence is starting and begin the scan.

\section{Data processing and viewing for sequence 6}

26. Obtain maximum intensity projections (MIP) or surface reconstructions of the nidus, feeding vessels, and draining veins, by using appropriate software.

Rule Out $(\mathrm{R} / \mathrm{O})$

Arteriovenous

Malformation

A1.5.6

On a GE system, one can use the Advantage Window Workstation with FuncTool version $1.9 M$. 
Table A1.5.7 Transverse Post-Contrast 3-D Spoiled Gradient Echo

\begin{tabular}{ll}
\hline Patient position & Supine \\
Scan type & 3-D spoiled gradient echo \\
Imaging plane (orientation) & Transverse \\
Variable bandwidth & Yes \\
Central slice or volume center & Nasion \\
Echo time $\left(T_{\mathrm{E}}\right)$ & Minimum full $(6 \mathrm{msec})$ \\
Receiver bandwidth $(\mathrm{RBW})$ & $15.6 \mathrm{kHz}$ \\
Repeat time $\left(T_{\mathrm{R}}\right)$ & $31 \mathrm{msec}$ \\
Flip angle $(\mathrm{FA})$ & $30^{\circ}$ \\
Fields of view $(\mathrm{FOV}, \mathrm{xOV}$ & $\mathrm{F})$ \\
Resolution $(\Delta x, \Delta y)$ & $240 \mathrm{~mm}, 180 \mathrm{~mm}$ \\
Number of data points collected $\left(N_{\mathrm{x}}, N_{\mathrm{y}}\right)$ & $0.93 \mathrm{~mm}, 0.93 \mathrm{~mm}$ \\
Display matrix $\left(D_{\mathrm{x}}, D_{\mathrm{y}}\right)$ & 256,192 \\
Slice thickness $(\Delta z)$ & 256,256 \\
Number of slices & $1.5 \mathrm{~mm}$ \\
Slice gap & 24 \\
Number of excitations $(\mathrm{NEX})$ & 0 \\
Number of acquisitions $\left(N_{\mathrm{acq}}\right)$ & 1 \\
Read direction & 1 \\
Flow compensation & Anterior-posterior \\
Extended dynamic range $(\mathrm{EDR})$ & Yes \\
Saturation pulses & Yes \\
Scan time & Inferior \\
\hline
\end{tabular}

\section{COMMENTARY}

\section{Background Information}

Catheter angiography remains the gold standard, particularly in the detection of small arteriovenous malformations, determining the feeding arteries, and detecting aneurysms and venous restriction. A 3-D spoiled gradient echo sequence without an inferior saturation pulse is similar to an MRA without magnetization transfer and has the additional advantage that whole-brain coverage can be obtained in a reasonable time (Fig. A1.5.1). A post-contrast 3-D spoiled gradient echo sequence (SPGR) often plays a significant role in treatment planning since it allows more accurate determination of the nidus shape and is therefore more tailored for treatment planning (Kondziolka et al., 1994; Schlemmer et al., 1994). Post-contrast 3-D SPGR is superior to noncontrast MRA in that it allows better visualization of the slower velocity draining veins (Yanno et al., 1997). Standard MRI in combination with post-contrast 3-D SPGR is also helpful in determining the degree of parenchymal involvement (Nussel et al., 1991). MIP reconstruction of the post-contrast 3-D SPGR data can prove helpful in visualizing the complexity of the arterial and venous vessels in relation to the AVM nidus (Fig. A1.5.2).

It is important to remember that other vascular malformations can look similar to arteriovenous malformations if the images are not closely evaluated. If a number of enlarged vessels are identified, but there is no definite nidus, a dural arteriovenous fistula is more likely than an arteriovenous malformation. Also, if a number of small veins are seen to drain into a larger anomalous vein, without arterial feeders, a developmental venous anomaly should be considered.

\section{Critical Parameters and Troubleshooting}

For a discussion of critical parameters and troubleshooting issues, see UNIT A1.2 \& A1.3.

In the evaluation of arteriovenous malformations, the inferior saturation band is not applied when acquiring the 3-D SPGR postcontrast, to allow inflow effect to improve vessel conspicuity.
Intracranial Arterial Disease

A1.5.7 


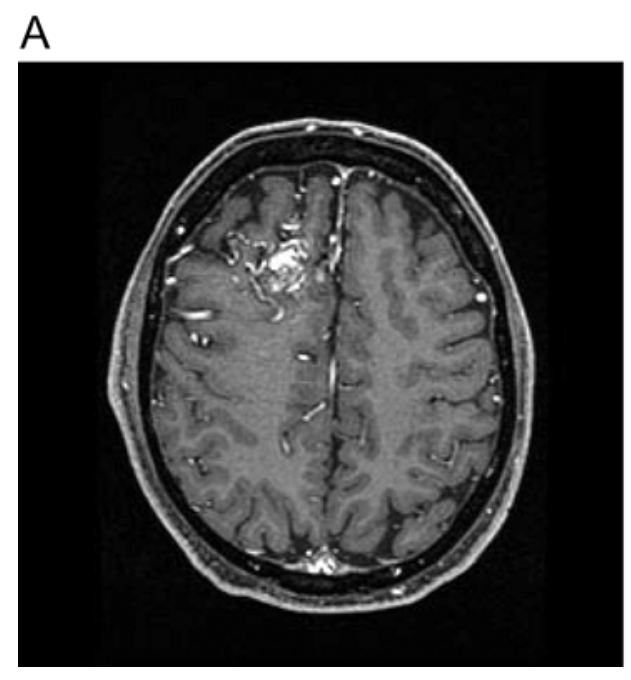

\section{B}

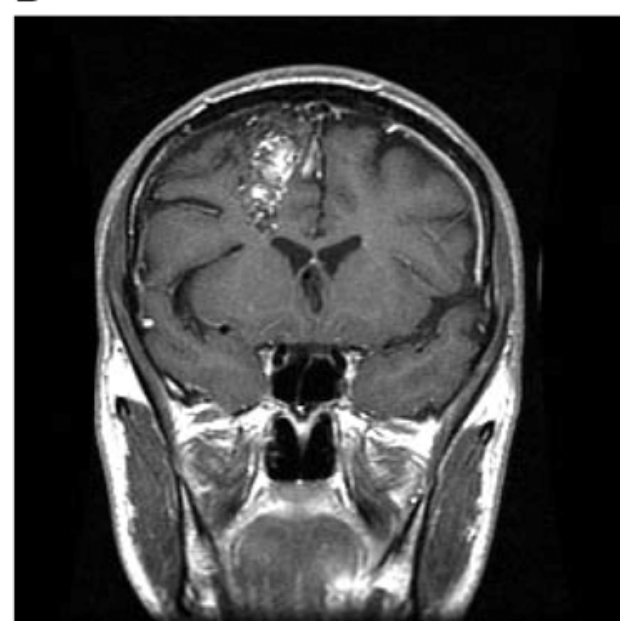

Figure A1.5.1 Post-contrast (A) transverse and (B) coronal 3-D gradient echo sequence demonstrating an arteriovenous malformaton (AVM) located in the right frontal lobe. The AVM is supplied by the right anterior and middle cerebral arteries, and is drained by enlarged cortical veins that drain into the superior saggital sinus.

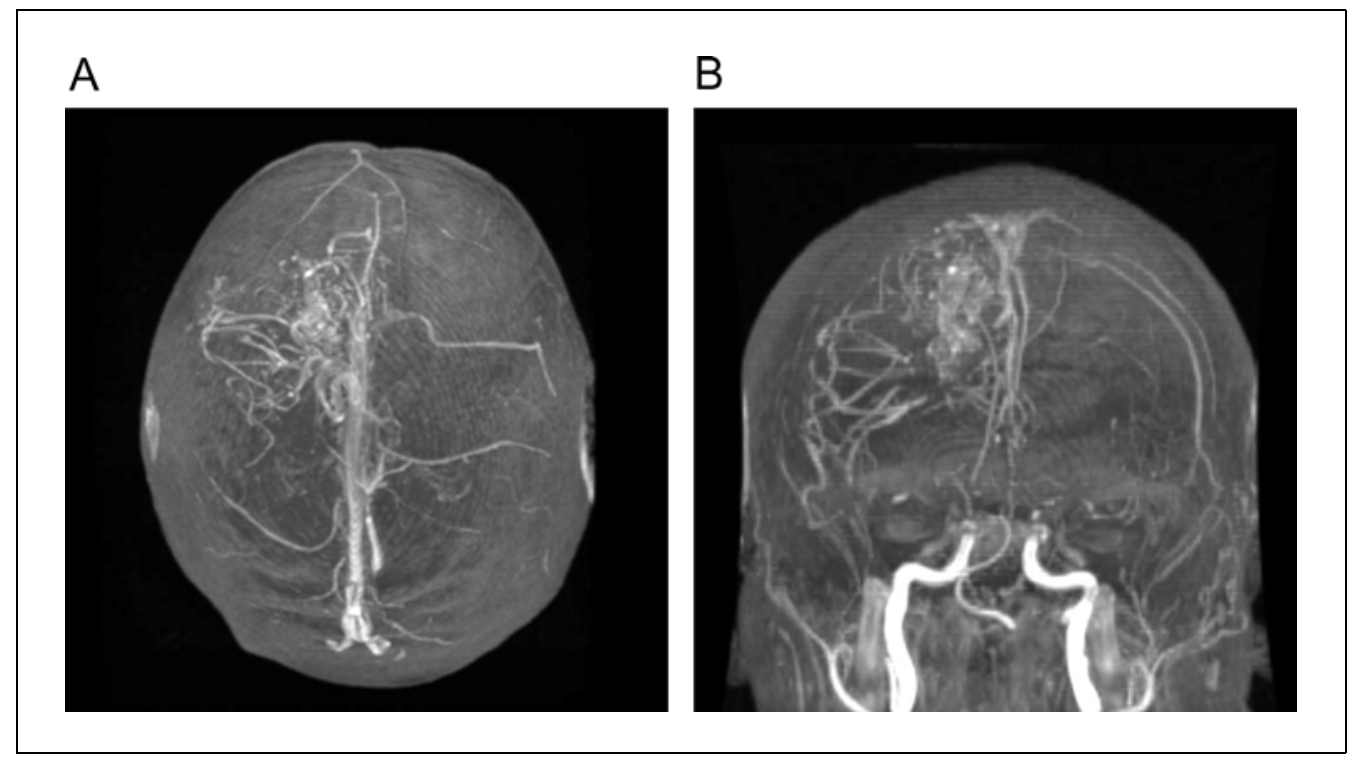

Figure A1.5.2 MIP images from the 3-D spoiled gradient echo data in (A) transverse and (B) coronal orientations. The feeding arteries from the anterior and middle cerebral arteries are demonstrated, as well as the enlarged cortical veins draining the AVM into the superior saggital sinus.

\section{Anticipated Results}

Post-contrast 3-D SPGR should allow accurate assessment of the size, shape, and location of AVM nidus with the caveat that a very small nidus can be missed on MR imaging. MRA can help in the assessment of potential arterial feeders and venous drainage routes and may help to identify aneurysms on feeding arteries or venous outflow restriction. However, catheter angiogram is still the gold standard for the detection of small AVMs, aneurysms, and venous restriction, and therefore catheter angiography may still be required. Standard imaging will allow assessment of the underlying brain parenchyma and determine if hemorrhage has occurred.

\section{Literature Cited}

Kondziolka, D., Lunsford, L.D., Kanal, E., and Talagala, L. 1994. Stereotactic magnetic resonance angiography for targeting in arteriovenous malformation radiosurgery. Neurosurgery 35:585590; discussion 590-591.

\section{A1.5.8}


Nussel, F., Wegmuller, H., and Huber, P. 1991. Comparison of magnetic resonance angiography, magnetic resonance imaging and conventional angiography in cerebral arteriovenous malformation. Neuroradiology 33:56-61.

Schlemmer, H.P., Hess, T., Debus, J., Knopp, M.V., Schad, L.R., and Engenhart, R. 1994. TOF-MR angiography in radiotherapy treated cerebral arteriovenous malformations. Radiologe 34:447453.

Shellock, F.G. 1996. Pocket Guide to MR Procedures and Metallic Objects. Lippincott-Raven, Philadelphia.

Yano, T., Kodama, T., Suzuki, Y., and Watanabe, K. 1997. Gadolinium-enhanced 3D time-offlight MR angiography. Experimental and clinical evaluation. Acta Radiol. 38:47-54.

Contributed by L. Kirkland Conrad, Steven Thibodeau, and Ellen Grant Massachusetts General Hospital

Boston, Massachusetts 